\title{
The nearby universe
}

DOI:

10.1093/astrogeo/atw103

\section{Document Version}

Final published version

Link to publication record in Manchester Research Explorer

\section{Citation for published version (APA):}

Argo, M., Beswick, R., Perez-Torres, M., \& Westcott, J. (2016). The nearby universe. Astronomy and Geophysics, 57(3), 3.36-3.39. https://doi.org/10.1093/astrogeo/atw103

\section{Published in:}

Astronomy and Geophysics

\section{Citing this paper}

Please note that where the full-text provided on Manchester Research Explorer is the Author Accepted Manuscript or Proof version this may differ from the final Published version. If citing, it is advised that you check and use the publisher's definitive version.

\section{General rights}

Copyright and moral rights for the publications made accessible in the Research Explorer are retained by the authors and/or other copyright owners and it is a condition of accessing publications that users recognise and abide by the legal requirements associated with these rights.

\section{Takedown policy}

If you believe that this document breaches copyright please refer to the University of Manchester's Takedown Procedures [http://man.ac.uk/04Y6Bo] or contact uml.scholarlycommunications@manchester.ac.uk providing relevant details, so we can investigate your claim.

\section{OPEN ACCESS}




\section{The nearby universe}

\author{
Megan Argo and colleagues \\ discuss what can be determined \\ from galactic observations and \\ surveys using e-MERLIN.
}

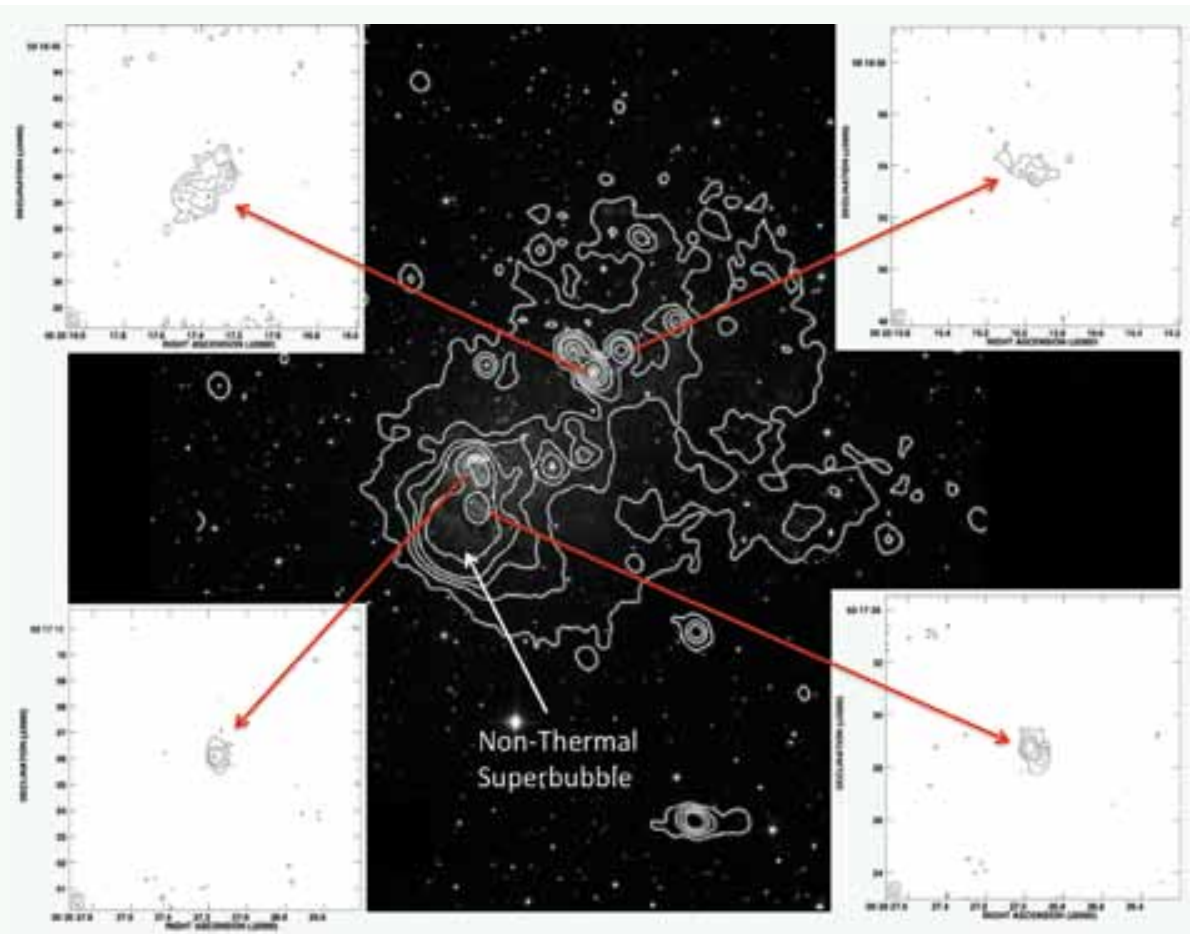

1 An image of dwarf galaxy IC10 showing the locations of four e-MERLIN detections. The background image is an H $\alpha$ map taken from the IPHAS survey (Barentsen et al. 2014), showing regions of recent star formation. The white contours show large-scale $20 \mathrm{~cm}$ emission (Heesen et al. 2015). The white boxes show high-resolution e-MERLIN contours of the detected compact sources and the red arrows show their location within IC10. (J Westcott [University of Hertfordshire])

Feedback from - and between - each of these processes is crucial to our understanding of galaxy evolution and appears to be essential if the observed galaxy luminosity distribution is to be reconciled with predictions from the standard hierarchical clustering models. Radio observations, both at high and low resolution, provide one of the best diagnostics of star formation and allow a direct view of the consequences of this process even in the most dusty regions toward the centre of galaxies.

\section{The dwarf galaxy IC10}

Dwarf galaxies are interesting from various astronomical standpoints, being both the most abundant type of galaxy in the local universe and the closest analogues to the very first galaxies that formed (Mateo 1998). They are relatively simple systems with stars forming in isolated regions, offering an ideal opportunity to study the effect of star formation on the surrounding environment in a low-density setting.
The best way to trace recent star formation is to look at massive stars. Stars themselves produce only a small amount of radio emission, but massive stars ionize the gas surrounding them to produce $\mathrm{HII}$ regions, large volumes of plasma that emit radio waves. At the end of its life, a massive star explodes as a supernova, injecting relativistic electrons into the surrounding gas. These electrons interact with magnetic fields in the gas, producing radio waves as they do so (Condon 1992). In the low-density environment of dwarf galaxies, however, these electrons can completely escape from the galaxy, locally reducing its levels of radio emission. The fraction of electrons that escape is currently unknown and, as a result, we do not fully understand how the "small-scale" radio emission from individual star-forming regions relates to the radio emission from an entire dwarf galaxy.

To answer these questions, a preliminary study of the nearby dwarf irregular galaxy IC10 has been carried out using uitous process in all galaxies that enriches the interstellar medium (ISM) of galaxies. 
2 The nearby starburst galaxy M82 seen in the optical (top left) and radio (bottom) with e-MERLIN at $1.4 \mathrm{GHz}$. The dashed box shows the location of the supernova SN 2014J, the middle inset shows the e-MERLIN view of the region, and the top inset shows that the explosion was also not detected with the European VLBI Network, to a limit of $400 \mu \mathrm{Jy} /$ beam. (M Perez-Torres [IAA-CSIC], optical image from Digitised Sky Survey)

the new e-MERLIN system (Westcott et al. in prep.). This galaxy has recently undergone an intense period of star formation (Crowther et al. 2003), making it an ideal subject. IC10 contains several compact radio sources, both compact $\mathrm{HII}$ regions and supernova remnants (figure 1). So far, we have not found any star-forming products inside the non-thermal superbubble of IC10 (Yang \& Skillman 1993). Superbubbles are large cavities of ionized gas inside galaxy discs; they are thought to originate from the winds of massive stars and collections of supernovae (Krause et al. 2013). The superbubble in IC10 indicates a recent ( $<4$ million years ago) and violent burst of star formation there (Heesen et al. 2015).

The lack of star-forming products in this area shows that the current environment must have a very low gas density. There is not much gas for the supernovae to interact with until their shockwaves reach the outskirts of the superbubble. Taken together, these results will help us to understand the history and current environmental conditions in this part of the galaxy.

To find out how common these properties are among dwarf galaxies, we are now analysing several other nearby dwarf galaxies including NGC 1569 and NGC 2366.

\section{The archetypal starburst galaxy M82}

As their name suggests, starburst galaxies exhibit much higher rates of star-formation than dwarf or normal galaxies; a large fraction of the massive stars in the local universe form in such galaxies. These massive stars evolve quickly, producing significant populations of supernovae and supernova remnants, which can be used to probe the current star-formation rate, supernovae astrophysics and the environment of the ISM. While significant star formation occurs in the centre of these galaxies, the high level of dust and gas obscures much of this star formation from view, so radio interferometry is vital in order to probe these turbulent regions.

The nearby starburst galaxy M82 provides a unique opportunity to study the detailed physical processes behind star formation and its consequences for the
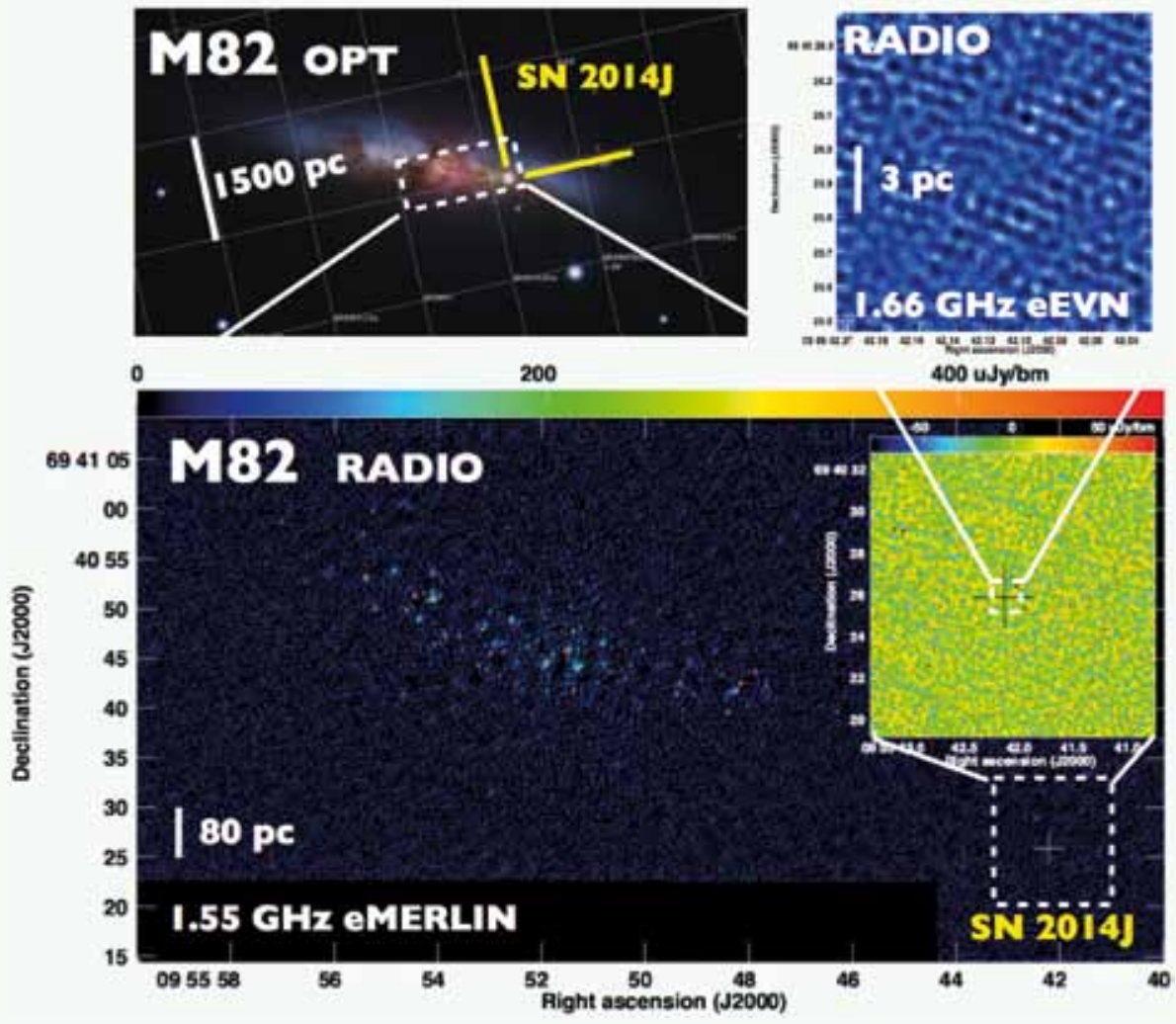

environment around a star-forming region. Within a kiloparsec radius, extended nonthermal and thermal radio emission traces recent star formation. At high angular resolution with e-MERLIN, this radio emission can be resolved into more than 50 compact radio sources: the majority $(\sim 2 / 3)$ are supernova remnants, the rest are a mixture of Hir regions and other sources related to star formation and accretion processes (Muxlow et al. 1994, Fenech et al. 2010, Gendre et al. 2013).

The enhanced sensitivity and image fidelity of e-MERLIN have almost doubled the number of compact radio sources that can be detected. The latest observations at $1.4 \mathrm{GHz}$ have revealed several new and unexpected structures, such as large linear shock features up to a few tens of parsecs long (shown on the cover of this issue). The true nature of these linear components is yet to be fully understood, although it is likely that they are similar in nature and size to those found within the centre of the Milky Way (Yusuf-Zadeh et al. 1999).

Deep 4.5-7.5GHz e-MERLIN observations have yielded the most sensitive image of this starburst to date, shown on the cover of this issue, imaging and resolving all of the sources in the central region with sensitivities of $\sim 10 \mu \mathrm{Jy}$. This resolution and sensitivity will allow the imaging of knot structures within all but the most compact of the radio supernova remnants. It will also provide adequate resolution to track the spatial extent of unique new sources, such as the candidate microquasar discovered in 2010 (Muxlow et al. 2010).

Studies of this nearby starburst galaxy are continuing with a series of new observations with e-MERLIN in combination with global VLBI arrays, using the new scope to correlate multiple e-MERLIN telescopes as part of the European VLBI Network to trace the evolution of the corecollapse supernovae as their shock fronts expand at $\sim 10000 \mathrm{~km} \mathrm{~s}^{-1}$. As well as measuring the motion of individual shock fronts, this current campaign is also searching for new radio supernovae and other transients. This is a long-term study that is bringing a better understanding of the physics of supernovae, their remnants and the ISM into which each of these sources expands.

\section{Thermonuclear supernovae}

There are two main classes of supernova explosion. Core-collapse supernovae mark the ends of single massive stars; they are often strong radio emitters because the shock front expands into the dense material of the surrounding interstellar medium, accelerating electrons. Type Ia supernovae, in contrast, are the result of a thermonuclear explosion of a white dwarf with a mass approaching the Chandrasekhar limit. These supernovae are useful as cosmological distance indicators and contribute significantly to the chemical evolution of galaxies, but the physics of these explosions is not understood in detail. This lack of knowledge makes modelling them difficult, compromising their use as distance indicators and adding a large uncertainty 

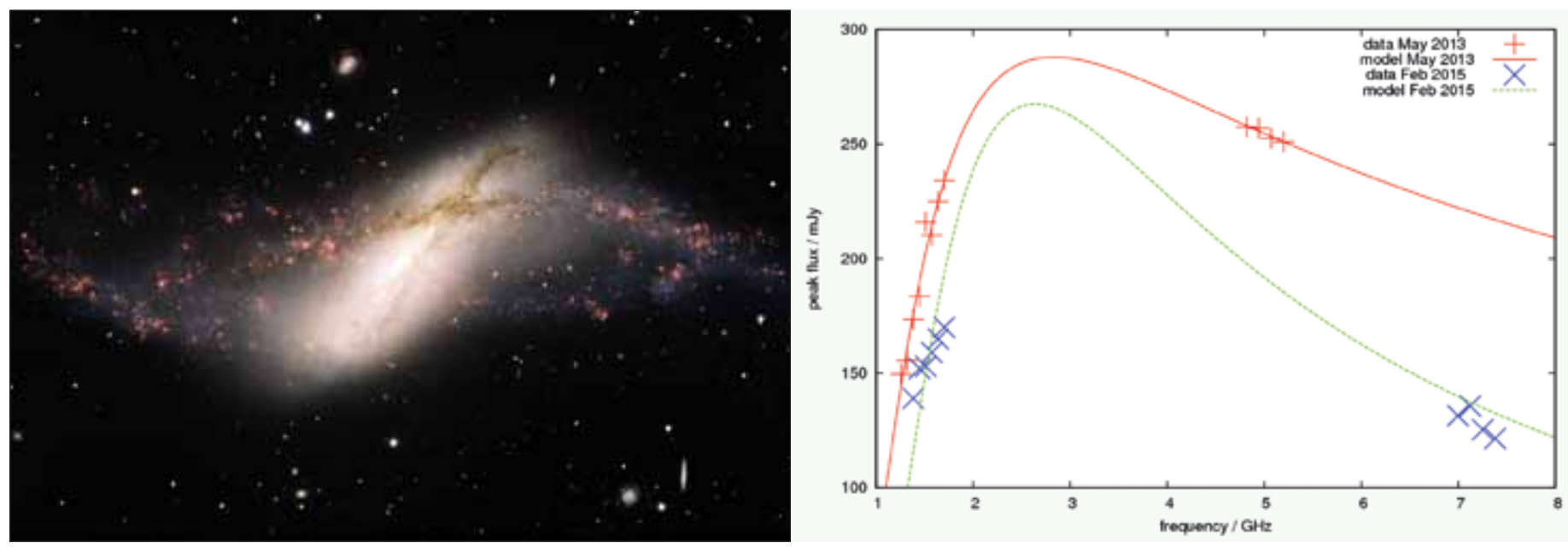

3 Left: A composite optical image of the polar ring galaxy NGC660, made using the Gemini Multi-Object Spectrograph on the Frederick C Gillett Gemini North Telescope, in August 2012. (Gemini Observatory/AURA). Right: The radio spectral energy distribution of the new radio source in the core of NGC660, from e-MERLIN observations taken in 2013 (red) and 2015 (green). (Argo et al. 2015)

to our understanding of their role in the changing composition of galaxies.

There are two basic models of the processes leading to a Type Ia supernova. In the single-degenerate (SD) scenario, a white dwarf accretes mass from a hydrogen-rich companion star, growing to reach a mass close to the Chandrasekhar mass and then exploding as a supernova. In the double-degenerate (DD) scenario, two white dwarfs merge; the more massive star disrupts then accretes its lower-mass companion, again exploding because it reaches a mass that is unstable.

Radio observations have the potential to discriminate between these two models. In all scenarios with mass transfer from a companion, a significant amount of circumstellar gas is expected, resulting in significant radio emission as shock fronts meet the gas. In contrast, the DD scenario is not expected to give rise to any nearby circumstellar medium; hence essentially no radio emission is expected. The recent supernova SN2014J in M82 brought a unique opportunity to test these proposed theoretical models.

Soon after its optical discovery, SN 2014J was observed with e-MERLIN and the European VLBI Network (EVN). This was the deepest ever search for a Type Ia supernova, but no radio emission was detected (figure 2). The lack of a detection excludes completely symbiotic SD systems and the majority of the models with stable nuclearburning white dwarfs, strongly favouring DD scenarios in which both stars are white dwarfs (van der Wel et al. 2014).

\section{A newly awoken black hole}

Active galaxies come in a variety of different flavours, but the activity is usually powered by either large amounts of star formation (as in M82), an active central

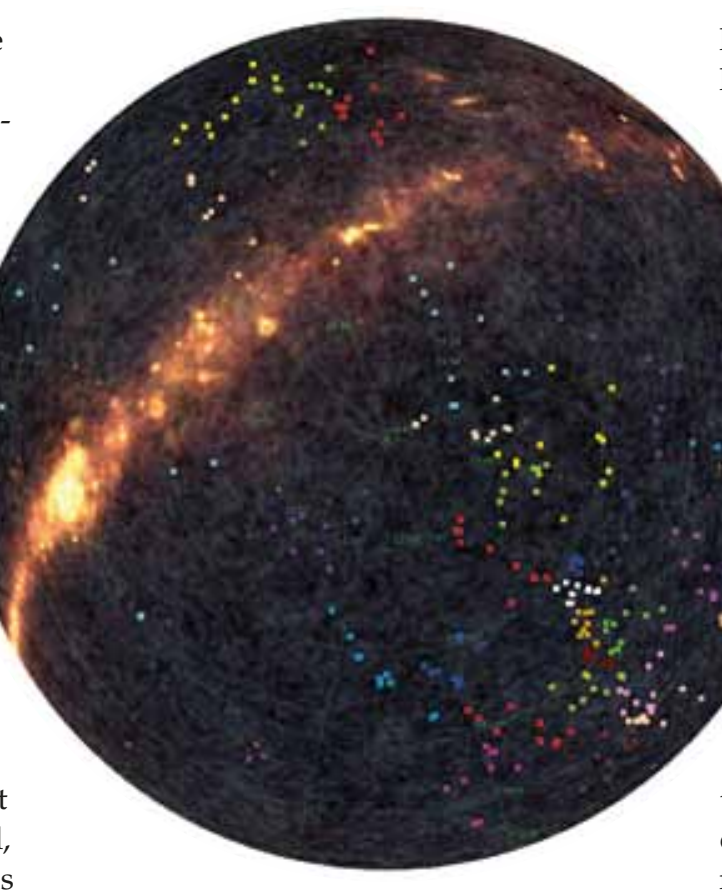

4 LeMMINGs sample, showing locations of all 280 galaxies. Colours represent different scheduling blocks. (M Argo [JBCA, University of Manchester])

supermassive black hole, or some combination of the two. Almost every galaxy, including our own, appears to have a black hole at its core. Most of the time these are quiet, with just their invisible gravitational pull shaping their surroundings, but in about $10 \%$ of galaxies the central black hole is much more active, swallowing material and spitting out giant jets. Radio-loud galaxies with active black holes are common in the high-redshift universe, but local examples of such activity are less common. So when a relatively nearby galaxy suddenly becomes active, there is much to be gained by watching the event at high resolution to see how the outburst develops on spatial scales impossible to probe in more distant systems.

One recent example is the nearby galaxy NGC660 (figure 3). Like most galaxies, it has a thick disc of stars and gas, but it also hosts a less dense polar ring of stars and smaller star-forming clouds, thought to be the remnants of a past encounter with another galaxy. Located just 42 million light years away, the galaxy contains a previously dormant black hole that is now becoming active as material falls onto it, probably as the end result of this galactic close encounter. The onset of such activity is rarely seen, so NGC660 provides an exciting opportunity to study an example in the neighbourhood of the Milky Way.

In 2012, astronomers noticed that NGC 660 had become hundreds of times brighter over just a few months; the average brightness of a normal galaxy tends to be very stable. Archive radio observations of the galaxy show a bright new radio source appeared in the centre of the galaxy in 2010 (Minchin et al. 2013). Both e-MERLIN and the EVN have since been used to investigate and monitor the activity in the core of this galaxy. Very high resolution images from the EVN show a very bright radio source at the dynamical centre of NGC 660, and evidence of jets moving at a substantial fraction of the speed of light. The new results from e-MERLIN, taking advantage of the much larger frequency coverage on the upgraded system, show that the object has a radio spectrum that peaks at around $2 \mathrm{GHz}$, exactly the sort of spectrum expected for a very young black hole jet system (figure 3).

The big question is whether the material in the jets has enough energy to overcome gravity and punch its way out of the galaxy, or if it will lose energy and fizzle out before getting that far. Studying the jet will give astronomers a clue about the initial eruption of the jet, and how much material fell onto the black hole to cause the outburst in the first place. 


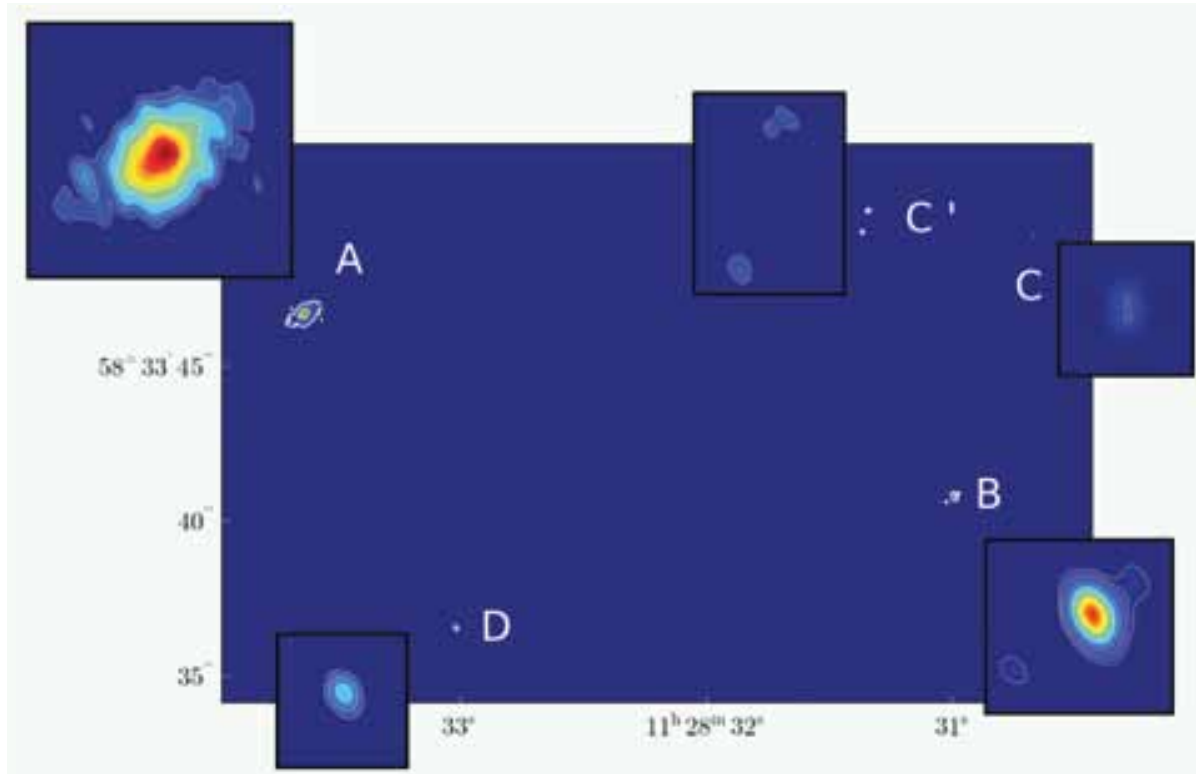

5 The starburst/low-luminosity AGN composite system Arp299, imaged by e-MERLIN as part of LIRGI.

\section{Surveys of nearby galaxies}

Within the e-MERLIN legacy programme, there are two projects undertaking very large surveys of nearby galaxies: LeMMINGs is considering local galaxies of all types, and LIRGI is taking a sample of extreme star-forming galaxies (see table 1 on page 3.29). In combination, these projects are aiming to chart the star formation and accretion activity across a range of galaxy types, from local dwarf galaxies through normal spiral galaxies like our own, out to galaxies in which some of the most intense star formation in the local universe is taking place.

The formation of stars is fundamental to the evolution of galaxies; accretion onto supermassive black holes provides a major power source in the universe, dominating the emission from distant quasars. These two legacy programmes aim to trace and accurately characterize the roles and interrelation of these two processes by examining the feedback between them down to subparsec scales in the local universe.

LeMMINGs uses a two-tiered approach to investigate star formation and accretion processes in $\sim 300$ nearby galaxies, covering all galactic environments from dwarf galaxies to massive ellipticals and active starbursts (figure 4). The most sensitive of these observations will image radio sources brighter than $\sim 10^{16} \mathrm{WHz}^{-1}$, detecting all radio supernova remnants brighter than
Cassiopeia A to a distance of $20 \mathrm{Mpc}$. This will provide the first systematic radio catalogue of individual star-formation indicators, resulting in a direct and obscuration-free picture of star formation and low-luminosity accretion in the local universe.

The LIRGI project will study a sample of local galaxies with significant infrared luminosity, tracing star formation in the most extreme starburst environments (figure 5). These galaxies are the nearby ........ analogues of the luminous high-redshift star-forming galaxies studied at higher redshift. A significant uncertainty in deriving the star-formation history of

the universe relates to whether the stellar initial mass function (that is, the distribution of stellar masses) becomes top-heavy in the intense star-formation environments that appear to be more common in the early universe.

One way to investigate this using nearby sources is via the identification of powerful core-collapse radio supernovae, the explosive end-results of massive stars. Using the sensitivity of e-MERLIN, modelling predicts that the survey will find about 10 detectable radio supernovae in $5 \mathrm{GHz}$ images, assuming starburst-like Arp 220like behaviour, and only about two if the IMF is more like that of the Milky Way. LIRGI observations are providing the sensitivity to measure the spectral curvature of emission across a wide bandwidth and so constrain free-free absorption arising from thermal gas, as well as mapping the largescale magnetic properties of galaxies using Faraday rotation and polarization. This will lead the way in the study of galactic magnetic fields, one of the prime SKA key science areas.

Detection of weak radio emission from AGN cores in the presence of the confusing diffuse emission from a starburst will depend on the fact that the core emission is linearly polarized, while the Faradaythick starburst emission is not. Using rotation measure (RM) synthesis, which involves making separate images for different assumed rotation measure values, we can pick out the polarized AGN core. This method also allows us to measure, simultaneously, the foreground RM, which holds information about galactic magnetic fields. A signal-to-noise ratio of 10 is needed to detect the RM over the large range of possible values, assuming a 10 polarized $0.5 \mathrm{mJy}$ central AGN, yielding a minimum sensitivity of $5 \mu \mathrm{Jy} /$ beam; this necessitates including the Lovell Telescope in the network. The resulting RM values, in conjunction with electron densities derived from free-free absorption, will be used to estimate field strengths and decide between competing weak and strong (dynamically significant) magnetic field models in ultraluminous LIRGs (Thompson et al. 2006).

An exciting new possibility is to use Zeeman splitting observations or line Faraday rotation in $\mathrm{OH}$ megamasers to measure magnetic field strengths in situ, and so discriminate between weak and strong field models (Robishaw et al. 2008). Detected sources have apparent circular polarization signals of $\sim 5 \mathrm{mJy}$. These e-MERLIN observations, which would require using the iconic $76 \mathrm{~m}$ Lovell Telescope as part of the network to boost sensitivity, will reach the thermal noise limit of $1 \mathrm{mJy}$ over the $50 \mathrm{kHz}$ channel width required to detect the splitting; without including the Lovell Telescope, detecting it would be impossible. Studies of $\mathrm{OH}$ using the new e-MERLIN correlator will for the first time allow all four $\mathrm{OH}$ transitions to be observed simultaneously, essential for imaging the weak satellite 1612 and $1720 \mathrm{MHz}$ transitions that can be used to constrain the maser pumping scheme and refine estimates of source properties (Lockett \& Elitzur 2008).•

\section{AUTHORS}

Megan Argo, Rob Beswick, Jodrell Bank

Observatory, University of Manchester, UK. Miguel

Perez-Torres Instituto de Astrofísica de Andalucía

(IAA-CSIC), Granada, Spain. Jonathon Westcott, University of Hertfordshire, UK.

\section{ACKNOWLEDGMENTS}

e-MERLIN is a National Facility operated by the
University of Manchester at Jodrell Bank Observatory on behalf of STFC.

\section{REFERENCES}

Argo MK et al. 2015 Mon. Not. R. Astron. Soc. 452 1081

Condon JJ 1992 Ann. Revs. Astron. Astrophys. 30575 Crowther PA et al. 2003 Astron. \&Astrophys. 404483 Barentsen G et al. 2014 Mon. Not. R. Astron. Soc.

\section{0}

Fenech D et al. 2010 Mon. Not. R. Astron. Soc. 408607

Gendre M A et al. 2013 Mon. Not. R. Astron. Soc. 4311107

Heesen V et al. 2015 Mon. Not. R. Astron. Soc. 447 LI Lockett P \& Elitzur M 2008 Astrophys. J. 677985 Mateo ML 1998 Ann. Rev. Astron. Astrophys. 36435 Minchin RF et al. 2013 AAS Meeting 221 (abstract)
Muxlow TWB et al. 1994 Mon. Not. R. Astron. Soc. 266455

Muxlow TW B et al. 2010 Mon. Not. R. Astron. Soc. 404 L109

Pérez-Torres M et al. 2014 Astrophys. J. 79238 Robishaw T et al. 2008 Astrophys. J. 680981 Thompson TA et al. 2006 Astrophys. J. 645186 van der Wel A et al. 2014 Astrophys. J. 792 L6 Yusuf-Zadeh F et al. 1999 Astrophys. J. 527172 